\title{
Role of Foley's Catheter in Removing Foreign Body of Oesophagus
}

\author{
Adhikari P, ${ }^{1}$ Bhusal CL, ${ }^{1}$ Guragain RPS, ${ }^{1}$ Acharya S, ${ }^{2}$ Budhathoki B' \\ 'Department of ENT and Head and Neck Surgery, ${ }^{2}$ Department of Anaesthesia, TU Teaching Hospital, Kathmandu, Nepal.
}

\begin{abstract}
Oesophageal foreign bodies are commonly removed with rigid oesophagoscopy under general anaesthesia, but spherical foreign bodies can also be removed using a foley's catheter under fluoroscopic control without anaesthesia. We present a case of successful removal of a spherical marble from the oesophagus in a four years old girl by a foley's catheter under general anaesthesia.
\end{abstract}

Keywords: Foreign body, oesophagus, foley's catheter

\section{INTRODUCTION}

Foreign body ingestion is one of the common otorhinolaryngologic emergency conditions. Usually, most oesophageal foreign bodies in children have been removed with rigid oesophagoscopy under general anaesthesia. ${ }^{1}$ Although rigid oesophagoscopy and removal of foreign body remains the standard practice for virtually all sharp objects and complicated cases, alternative methods of extraction for smooth objects in an uncomplicated setting have been advocated in recent years. These include in patient or out patient observation, use of Magill's forceps, flexible oesophagoscopy, removal by foley's catheter, use of balloon catheter in conjunction with tooth forceps, oesophageal boungienage, forceps extraction, use of bougie to advance into the stomach and surgery apart from a few other innovative practices. ${ }^{2,3}$ Here, we report a case of spherical foreign body in the oesophagus removed by foley's catheter under general anaesthesia.

\section{CASE REPORT}

A four years old girl presented to the Department of Ear, Nose, Throat and Head and Neck Surgery of Tribhuvan University Teaching Hospital, Kathmandu with accidental ingestion of a marble. She had dysphagia but no history of respiratory distress. On examination child was drooling the saliva. X-ray soft tissue neck lateral view revealed a circular radio-opaque foreign body of size $1 \mathrm{~cm}$ by $1 \mathrm{~cm}$ at the level of $\mathrm{C}_{6}$ $\mathrm{C}_{7}$ (Figure 1). Rigid oesophagoscopy was performed and conventional forceps was used to hold the foreign body but it slipped off every time it was attempted. A 10 FG foley's catheter was passed down under the supervision of direct laryngoscopy in the oesophagus below the marble and it was inflated with $3 \mathrm{ml}$ of normal saline. The catheter was pulled out slowly and the marble was extracted in hypopharynx from where it was removed successfully by a forceps (Figure 2). The child was asymptomatic after the procedure and discharged from hospital on the next day.
Correspondence:

Dr. Prakash Adhikari

Department of ENT and Head and Neck Surgery

TU Teaching Hospital, Kathmandu, Nepal

Email: prakash_ooz@hotmail.com

Phone: 9851015002 
Adhikari et al. Role of Foley's Catheter in Removing Foreign Body of Oesophagus

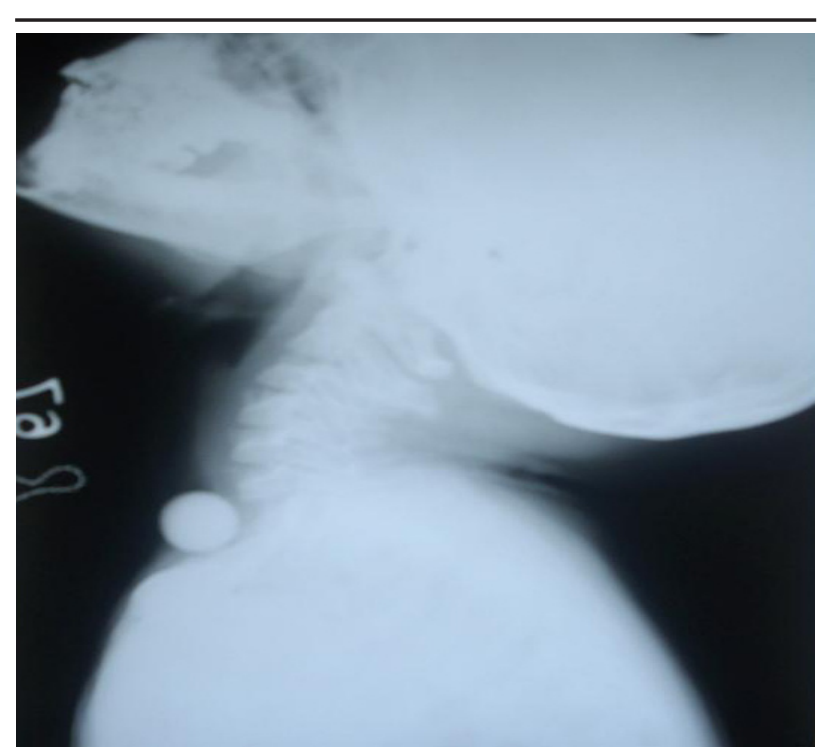

Figure 1. X-ray Soft tissue neck showing radio opaque foreign body at C6-7 level

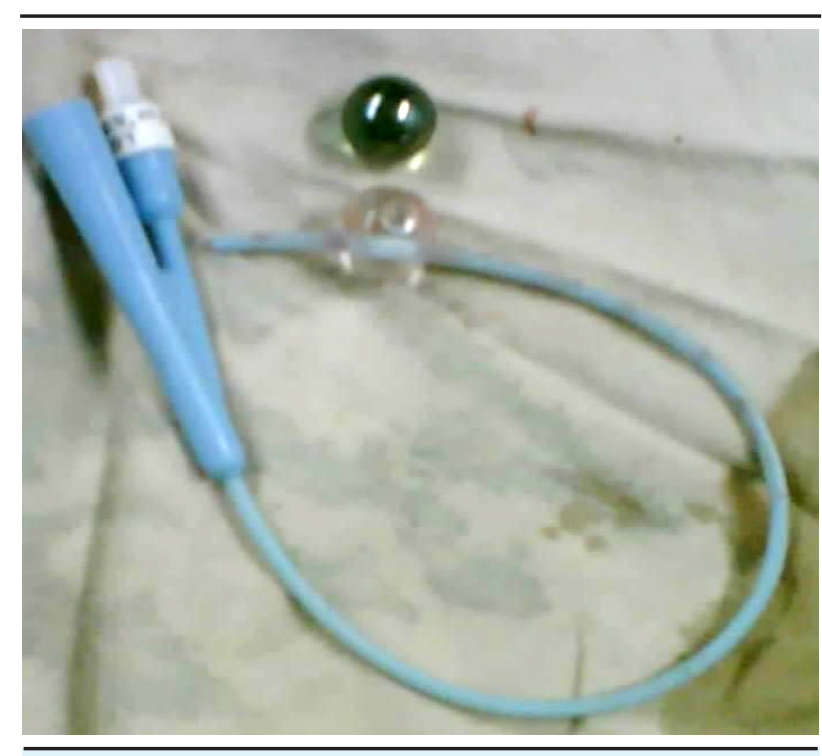

Figure 2. Marble and foley's catheter after extraction

\section{DISCUSSION}

Oesophageal foreign bodies in children are one of the most common emergencies that are frequently encountered in ENT practice. Coins are the most common foreign bodies in children. ${ }^{4}$ Foreign bodies in children mostly present at the level of cricopharyngeal junction. ${ }^{4}$

Spherical foreign bodies like marbles in the oesophagus are uncommon in our hospital too. Such foreign bodies are difficult to remove by rigid oesophagoscopy and conventional forceps. They require different techniques and instruments.

Foley's catheter has been used to remove such spherical foreign bodies in the west. Removal of foreign body by foley's catheter is usually conducted under fluoroscopic guidance and has been establish as a relatively safe and cost effective procedure. ${ }^{5,6}$ But in our case, as we did not have fluoroscopic control, we did direct laryngoscopy and insert foley's catheter using stone holding forceps. Foley's catheter is generally known for its usefulness in removing recently ingested and proximally located blunt objects. Foley's catheter extractions involve passing a balloon catheter distally to the ingested object, inflating the balloon and withdrawing the catheter and the ingested object proximally. It has been shown to be effective even without the usual sedation and fluoroscopic guidance and is recommended as the treatment of choice for retained coins in children who do not show signs of significant oesophgeal oedema that would causing tracheal impairment. However, there are certain contraindications for the use of foley's catheter technique, which include foreign body ingestion more than 24 hours before intervention or at an unknown earlier time, prior oesophageal stricture or surgery, signs and symptoms of marked oesophageal obstruction, stridor or compromised respiration. ${ }^{7}$

Besides foley's catheter, angiographic catheter has also been used by different authors. ${ }^{8}$ Foley's catheter seems to be an alternative technique to remove spherical and proximal foreign bodies from the oesophagus.

\section{REFERENCES}

1. Marrow SE, Bickler SW, Kennedy AP, Syndler CL. Baloon extraction of esophageal foreign bodies in children. J Pediatr Surg 1998;33:266-70.

2. Dunlap LB. Removal of an esophageal foreign body using a foley catheter. Am Emerg Med 1981;10:101-3.

3. Calkins CM, Christians KK, Sell LL. Cost analysis in the management of esophageal coins: endoscopy versus bougienage. J Pediatr Surg 1999;34:412-4.

4. Adhikari P, Shrestha BL, Baskota DK, Sinha BK. Accidental foreign body ingestion: analysis of 163 cases. Intl Arch Otorhinolaryngol 2007;267-70.
5. Conners GP. A literature based comparison of three methods of pediatric esophageal coin removal. Pediatr Emerg Care 1997;13:154-7.

6. Kelley JE, Leech MH, Carr MG. A safe and cost effective protocol for the management of esophageal coins in children. J Pediatr Surg 1993;28:898-900.

7. Campbell JB, Foley LC. A safe alternative to endoscopic removal of blunt esophageal foreign bodies. Arch Otolaryngol 1983;109:323-5.

8. Reddy NV, Bhatt C, Vaughan-Jones RH, Reddy TN. Spherical foreign bodies in the oesophagus removed by balloon angiographic catheter. J Laryngol Otol 2002;116:208-10. 